\title{
Experimental and theoretical studies on a novel helical architecture driven by hydrogen and halogen bonding interactions
}

\author{
QING ZHU LIU ${ }^{\mathrm{a}}$, SHAN SHAN WANG ${ }^{\mathrm{a}}$, TENG FEI WANG ${ }^{\mathrm{a}}$, JIAN GUO LIN ${ }^{\mathrm{a}, *}$, \\ XUE HAI JU ${ }^{\mathrm{b}}$ and LING QIU' ${ }^{\mathrm{a}, *}$ \\ ${ }^{a}$ Key Laboratory of Nuclear Medicine, Ministry of Health, Jiangsu Key Laboratory of Molecular Nuclear \\ Medicine, Jiangsu Institute of Nuclear Medicine, Wuxi 214063, People's Republic of China. \\ ${ }^{b}$ Institute for Computation in Molecular and Material Science, School of Chemical Engineering, Nanjing \\ University of Science and Technology, Nanjing 210094, People's Republic of China \\ e-mail: linjianguo@jsinm.org; qiuling@jsinm.org
}

MS received 1 February 2016; revised 19 September 2016; accepted 12 October 2016

\begin{abstract}
A novel two-dimensional (2D), layered, helical supramolecular architecture constructed via cooperative hydrogen bond and halogen bonds was synthesized and characterized: $\left[(\mathrm{BMBA})_{2}(\mathrm{TPB})\right]_{n}(\mathbf{1})[\mathrm{BMBA}=$ 3-bromo-2-methylbenzoic acid, TPB = 1,2,3,4-tetra-(4-pyridyl)-butane]. Density functional theory (DFT) calculations were carried out to investigate the nature of intermolecular interactions between BMBA and TPB. The cooperation between hydrogen bond and halogen bond in building up the open organic architecture was demonstrated elaborately. Complex 1 exhibits strong photoluminescence and high thermal stability. The nature of electronic transitions in the photoluminescent process was investigated by means of time-dependent DFT (TDDFT) calculations and molecular orbital analyses, revealing that the luminescent property of the helical supramolecular architecture of $\mathbf{1}$ was ligand-based. Periodic DFT calculations show that $\mathbf{1}$ is an electrical insulator with a band gap of $3.29 \mathrm{eV}$.
\end{abstract}

Keywords. Helical supramolecular architecture; crystal structure; hydrogen bond; halogen bond; photoluminescence.

\section{Introduction}

As is well-known, crystal engineering comprises of an understanding of specific intermolecular interactions, such as hydrogen bonding (HB), halogen bonding (XB), electrostatic interaction and van der Waals force, which govern the crystal packing and thus allow designing solids with tailored physical, chemical and biological properties. ${ }^{1-5}$ Therefore, they play a very important role in many fields as diverse as materials, medicine and interdisciplinary science, and hence become the subject of intense research interest from both experimental and theoretical points of view. Of all the intermolecular interactions, $\mathrm{HB}$ is the most common and important type of weak interaction. ${ }^{6}$ During the past decade, the $\mathrm{XB}$ interaction is also under active investigation, ${ }^{7-11}$ in parallel with those of $\mathrm{HB}$ in terms of strength and directionality. ${ }^{12}$

The highly selective, directional nature and appropriate strength make them ideal for use in the construction and stabilization of large noncovalently linked supramolecular architectures. ${ }^{4,13}$ Besides a plenty of

*For correspondence supramolecular architectures based on HB, many architectures based on XB have been intensively studied and can now be considered as a potential option in design and synthesis of new organic supramolecular systems with desired architectures and functions. $^{3,4}$ Recently, the competition between HB and $\mathrm{XB}$ in molecular assembly has been explored by some researchers. ${ }^{14-16}$ More importantly, effective supramolecular synthetic strategies around a hierarchy of architectures that comprise both $\mathrm{HB}$ and $\mathrm{XB}$ through systematic co-crystallization reactions were preliminarily developed and many high-dimensional molecularscale networks with attractive properties have been well documented. ${ }^{14-16}$ However, the recognition of HB and $\mathrm{XB}$-driven supramolecular architectures from experimental and theoretical points of view is still largely unexplored. ${ }^{17,18}$

It is known that with the rapid development of computational chemistry in the past decade, theoretical modelling of supramolecular chemistry has become more mature than ever. Many important chemical and physical properties of the chemical system can be predicted from various computational techniques. Density functional theory (DFT) has long been recognized as a 
better alternative tool in the study of organic chemical systems than the ab initio methods, because of the fact that it is computationally less demanding for inclusion of electron correlation. Moreover, DFT approaches have been demonstrated to be successful in helping to understand intermolecular interactions in many different systems. ${ }^{18-23}$

In the present paper, we report the synthesis, characterization, stability, fluorescence and thermodynamic properties of a novel 2D supramolecular architecture with $2_{1}$ helical system, $\left[(\mathrm{BMBA})_{2}(\mathrm{TPB})\right]_{n}(\mathbf{1})(\mathrm{BMBA}$ = 3-bromo-2-methylbenzoic acid, TPB $=1,2,3$,4-tetra(4-pyridyl)-butane, Scheme 1). This complex demonstrates that $\mathrm{HB}$ and $\mathrm{XB}$, which most often are seen in competition can also cooperate in building up open organic architectures. The nature of $\mathrm{HB}$ and $\mathrm{XB}$ interactions, dimerization, crystal packing and photoluminescence assignment were also investigated by DFT and TDDFT calculations. The experimental and theoretical structural studies will undoubtedly assist in developing versatile strategies for the assembly of highdimensional molecular architectures comprising both types of bonds.

\section{Experimental and Computational Methods}

\subsection{Materials and Methods}

All the reagents and solvents were commercially available and used as received. 2,3,4,5-Tetra-(4-pyridyl)thiophene was synthesized as reported ${ }^{24}$ and then reductively desulfurated to get the ligand 1,2,3,4-tetra(4-pyridyl)-butane (TPB, Scheme 1). ${ }^{25}$ The elemental analysis was carried out with a Perkin-Elmer 240C elemental analyzer. The IR spectrum was recorded using $\mathrm{KBr}$ pellets in the range of $4000-400 \mathrm{~cm}^{-1}$ on a Bruker Vector 22 spectrometer. ${ }^{1} \mathrm{H}$ and ${ }^{13} \mathrm{C}$ NMR spectra were recorded on a Bruker DRX-400 spectrometer at ambient temperature with tetramethylsilane (TMS) as internal reference. The powder X-ray diffraction
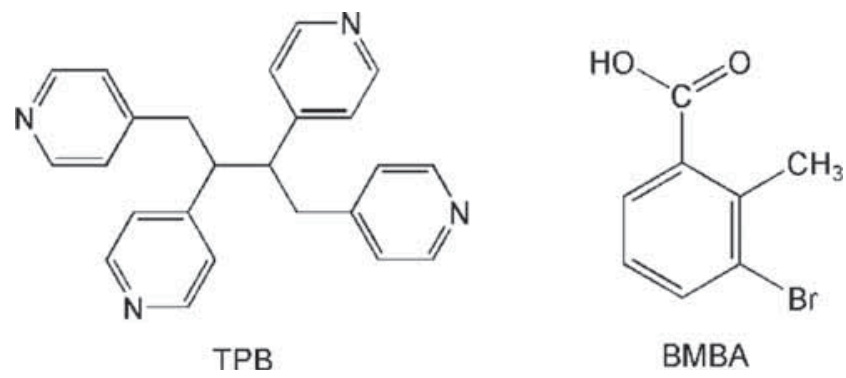

Scheme 1. Building blocks of the supramolecular architecture.
(PXRD) pattern was recorded on a RigakuD/maxRA rotating anode $\mathrm{X}$-ray diffractometer with graphite monochromatic $\mathrm{Cu} K \alpha(\lambda=1.542 \AA)$ radiation at room temperature. Thermogravimetric analysis (TGA) was performed on a simultaneous SDT 2960 thermal analyzer under flowing $\mathrm{N}_{2}$ with a heating rate of $10^{\circ} \mathrm{C} / \mathrm{min}$ from ambient temperature to $800^{\circ} \mathrm{C}$. Luminescence spectra for the solid sample of $\mathbf{1}$ were recorded with a Hitachi 850 fluorescence spectrophotometer.

\subsection{Synthesis of $\left[(B M B A)_{2}(T P B)\right] n(1)$}

Complex 1 was synthesized hydrothermally in a $23 \mathrm{~mL}$ Teflon-lined autoclave by heating a mixture of equal mole $(0.2 \mathrm{mmol})$ of BMBA $(0.0430 \mathrm{~g})$ and TPB $(0.0732 \mathrm{~g})$ in a $10 \mathrm{~mL}$ of water-ethanol $(1: 1 \mathrm{v} / \mathrm{v})$ solution at $120^{\circ} \mathrm{C}$ for $72 \mathrm{~h}$. After the mixture was slowly cooled to room temperature, light yellow block-shaped crystals were obtained. (yield: $48.6 \%$ ). M.p. $175^{\circ} \mathrm{C}$. Anal. Calc. (\%) for: $\mathrm{C}_{40} \mathrm{H}_{36} \mathrm{Br}_{2} \mathrm{~N}_{4} \mathrm{O}_{4}: \mathrm{C}, 60.31 ; \mathrm{H}, 4.56$; N, 7.03. Found (\%): C, 60.24; H, 4.71; N: 6.91. IR $\left(\mathrm{KBr}, \mathrm{cm}^{-1}\right): 3411 \mathrm{~s}, 3052 \mathrm{w}, 2426 \mathrm{~m}, 1955$ m, 1699 m, 1610 s, 1559 m, 1419 m, 1356 w, 1262 w, 1066 m, 1028 w, 1000 m, 938 w, 828 s, 758 m, 692 m, 629 w, $590 \mathrm{w}, 574 \mathrm{~m}, 539 \mathrm{~m}$.

\section{$2.3 X$ X-ray crystallographic study}

Single crystals of complex $\mathbf{1}$ in appropriate dimensions were collected for structure determination on a Bruker SMART APEX CCD diffractometer using graphitemonochromatized $\mathrm{Mo} / \mathrm{K} \alpha$ radiation $(\lambda=0.71073 \AA)$ at room temperature using the $\omega$-scan technique. Data reductions and absorption corrections were performed with the SAINT ${ }^{26}$ and SADABS ${ }^{27}$ software packages, respectively. The structures were solved by direct methods using the SHELXS-97 program $^{28}$ and were further refined by the full-matrix least-squares technique using the SHELXL-97 program. $^{29}$ All the non-hydrogen atoms were refined with anisotropic displacement parameters, and hydrogen atoms were generated geometrically with assigned isotropic thermal parameters. Analytical expressions of neutral-atom scattering factors were employed, and anomalous dispersion corrections were incorporated.

\subsection{Computational details}

Two possible dimers and related monomers obtained from the crystal structure were used to calculate and evaluate their geometric and electronic properties (Figure S1 in Supplementary Information). Dimer I consists of an intermolecular hydrogen bond O1-H1A...N1 (The symmetry code for \#A is " $1-\mathrm{x}$, 
$-\mathrm{y}, 2-\mathrm{z}$ "), while dimer II consists of a halogen bond $\mathrm{C} 16-\mathrm{Br} 1 \ldots \mathrm{N} 2$. Geometry optimizations were carried out using hybrid DFT method at the MPW1PW91 (modified Perdew-Wang exchange functional and Perdew-Wang 91 correlation functional) ${ }^{30}$ level with the 6-31G** basis set. ${ }^{31}$ Geometries were fully optimized without any symmetry restriction by the Berny method. To characterize the nature of stationary point and to determine the zero-point vibrational energy (ZPVE) correction, harmonic vibrational analysis was performed subsequently on each optimized structure at the same level. According to the previous studies, the DFT computed harmonic vibrational frequencies are usually larger than those observed experimentally; so, the scale factor of 0.96 was used uniformly to take into account the systematic overestimation of vibrational frequencies in the MPW1PW91/6-31G** calculations. ${ }^{32}$ Based on the scaled harmonic frequencies thermodynamic properties and expected changes upon dimerization were derived from the principles of statistical thermodynamics. ${ }^{33}$ The interaction energies among the complex were evaluated from the energy differences between the dimers and the monomers. The basis set superposition error (BSSE) was determined for the interaction systems using the standard counterpoise correction method of Boys and Bernardi. ${ }^{34}$ To verify the suitability of the basis set used, single-point calculations were further carried out for all the dimers with the $6-311++\mathrm{G}(3 \mathrm{df}, 3 \mathrm{pd})$ basis set. ${ }^{35,36}$ All calculations concerning the gas phase were performed with the Gaussian09 program. ${ }^{37}$

The crystal structure determined by X-ray diffraction was used for the computation of bulk properties, including the electronic band structure, density of states, and optical properties. ${ }^{38}$ The calculations were performed using the DFT method with Vanderbilttype ultrasoft pseudopotentials ${ }^{39}$ and a plane-wave expansion of the wave functions as implemented in the CASTEP code. ${ }^{40}$ The generalized gradient approximation (GGA) functional proposed by Perdew, Burke and Ernzerhof (PBE) ${ }^{41}$ was employed and the cutoff energy of plane waves was set to $260.0 \mathrm{eV}$. The electronic wave functions were obtained by the Pulay density-mixing scheme ${ }^{42}$ and Brillouin zone sampling was performed by using the special Monkhost-Pack scheme. ${ }^{43}$

\section{Results and Discussion}

\subsection{Synthesis and characterization}

Complex 1 was synthesized under the hydrothermal reaction from the corresponding precursors, resulting

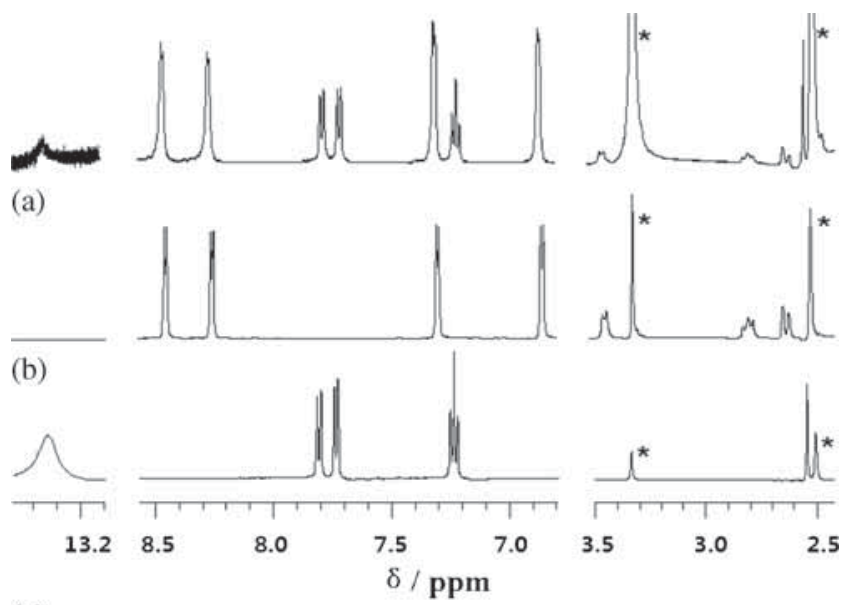

(c)

Figure 1. ${ }^{1} \mathrm{H}$ NMR spectra of complex 1. (a) TPB; (b) and BMBA; (c) in DMSO. *indicates the signal of the solvent.

in pale yellow block crystals. It is soluble in polar solvents such as DMSO and characterized by the NMR spectroscopy preliminarily. An excellent agreement is exhibited in the ${ }^{1} \mathrm{H}$ NMR spectra of the complex $\mathbf{1}$ and the ligands, indicating absence of any impurity (Figure 1). Also, the chemical structure of the complex was verified by ${ }^{13} \mathrm{C}$ NMR spectrum (Figure S2, in Supplementary Information). During self-assembly of the complex from the ligands, only a broad signal at high magnetic field was observed that was assigned to the weakened proton of $\mathrm{O}-\mathrm{H}$ in the ligand BMBA. This may be attributed to the formation of intermolecular hydrogen bonding. Moreover, from the integral analysis of ${ }^{1} \mathrm{H}-\mathrm{NMR}$, it can be found that $\mathbf{1}$ was formed by BMBA and TPB in a ratio of 2:1. The powder Xray diffraction (PXRD) was used to check the phase purity of the bulk sample in the solid-state. As shown in Figure 2, the main diffraction peaks of the PXRD for the as-synthesized sample have been indexed. The reflection positions and relative intensity of the measured PXRD pattern closely match the simulated pattern generated from the result of single crystal diffraction, indicating that it is a pure product.

\subsection{Crystal structure and optimized structure}

X-ray diffraction study reveals that complex $\mathbf{1}$ crystallizes in a centrosymmetric monoclinic space group $P 2_{1} / n$ (Table $\mathrm{S} 1$ in Supplementary Information), and the asymmetric unit contains one BMBA and half TPB (Figure 3a). Noteworthy fact is that TPB contains four equivalent discrete $\mathrm{N}$-donor sites with approximate bond angles of $53.9^{\circ}$ and $126.1^{\circ}$ between two adjacent $\gamma$-methylpyridine and four $\mathrm{N}$-donor atoms in a plane. So, it can behave as both HB- and XB-acceptor sites. 


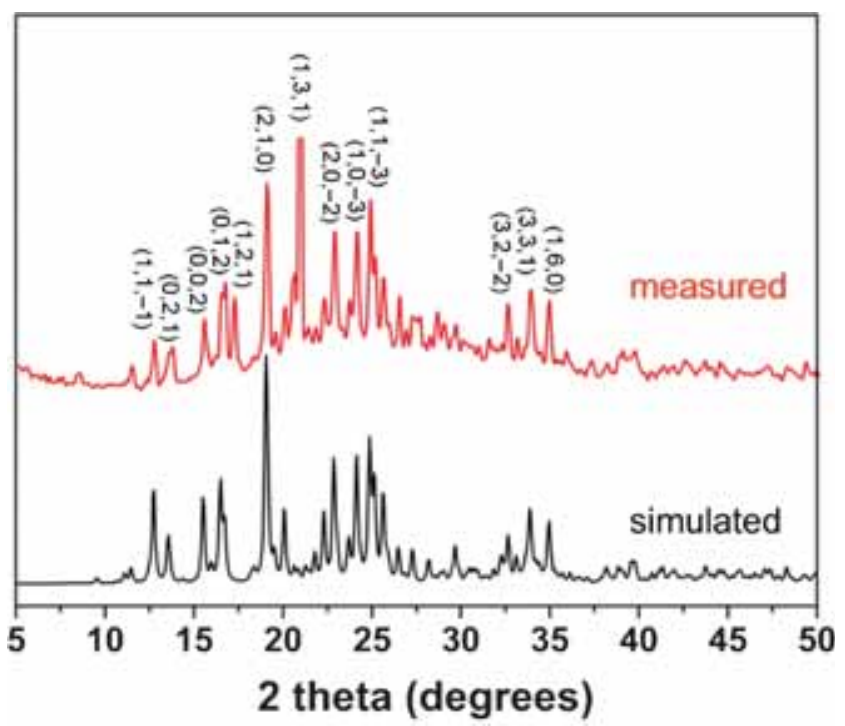

Figure 2. The simulated and measured PXRD patterns of complex 1.

As for the ligand BMBA, it also has two sites for potentially binding processes and therefore it is also a potential candidate to be engaged in simultaneous $\mathrm{HB}$ and XB. As anticipated, there are two kinds of intermolecular interactions between BMBA and TPB in the supramolecular architecture (Figure $3 b$ ). One is the typical hydrogen bond $\mathrm{O}-\mathrm{H} \cdots \mathrm{N}[\mathrm{O} \cdots \mathrm{N} 2.5908(4) \AA$, $\left.\mathrm{H} \cdots \mathrm{N} 1.7790(3) \AA, \angle \mathrm{O}-\mathrm{H} \cdots \mathrm{N} 170.209(18)^{\circ}\right]$, and the other is the attractive halogen bond $\mathrm{C}-\mathrm{Br} \cdots \mathrm{N}$

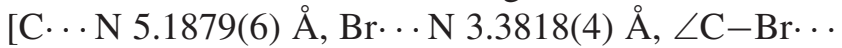
$\left.\mathrm{N} 160.664(7)^{\circ}\right]$. The halogen bond is only slightly shorter than the sum of the van der Waals radius $(3.44 \AA),{ }^{44}$ indicating that it is a weak interaction. The reported molecular architectures assembled by $\mathrm{C}-\mathrm{Br} \cdots \mathrm{N}$ intermolecular interactions with $\mathrm{Br} \cdots \mathrm{N}$ distances in the range of $3.192(4)-3.324(3) \AA$ and $\mathrm{C}-\mathrm{Br} \cdots \mathrm{N}$ angles ranging from $160.17(11)$ to $172.38(13)^{\circ}$ were observed..$^{38}$ The geometrical parameters for the halogen bond found in our study are comparable to the reported $\mathrm{Br} \cdots \mathrm{N}$ contacts. This $\mathrm{XB}$ interaction is not neglected, while $\mathrm{C}-\mathrm{Br} \cdots \mathrm{N}$ seems to cooperate with strong hydrogen bond $\mathrm{O}-\mathrm{H} \cdots \mathrm{N}$ in our investigated structure. Therefore, the bond length and angle of halogen bond may be influenced by the primary source of halogen bond. This was further demonstrated by DFT calculations on possible dimers of TPB and BMBA, which were inferred from the crystal structure of complex 1 which consisted of one $\mathrm{HB}$ and one $\mathrm{XB}$ $(\mathbf{I}, \mathrm{O}-\mathrm{H} \cdots \mathrm{N}$; II, C-Br $\cdots \mathrm{N}$ ), respectively (Figure S1).

Propagation of such hydrogen and halogen bonding interactions leads to the formation of 2D helical architecture (Figure 4), demonstrating the feasibility of such a structure entirely due to noncovalent interactions. By closer inspection, one BMBA and half TPB are found to assemble by alternative intermolecular interactions to give an infinite cylindrical $2_{1}$ helix through spontaneous assembly. The other half TPB molecule is assembled in the neighboring helix. The helical pitch, given by one full rotation around the $2_{1}$ helical axis, is 15.939 (3) $\AA$. The left- and right-handed helixes are alternately arranged to make the whole architecture racemic. The distance between the repeating parts of the helical chains is $c a$. $15.2 \AA$ and the size of the helical channel is ca. $11.1 \times 12.8 \AA$. Based on our understanding of supramolecular architectures, this is the first report on the $2 \mathrm{D}$ open organic architecture with a racemic helical system driven by mixed $\mathrm{HB}$ and $\mathrm{XB}$.

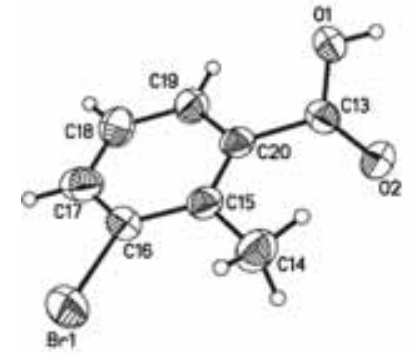

(a)

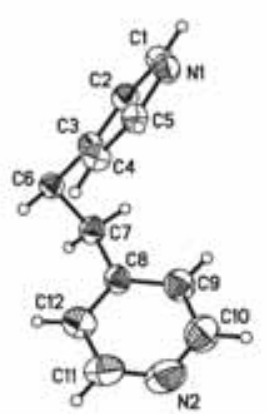

Figure 3. (a) The asymmetric unit of complex $\mathbf{1}$ with atomic displacement ellipsoids drawn at the $50 \%$ probability level. (b) Intermolecular interactions consist of $\mathrm{O}-\mathrm{H} \cdots \mathrm{N}[\mathrm{O} \cdots \mathrm{N} 2.5908(4) \AA, \mathrm{H} \cdots \mathrm{N}$ $\left.1.7790(3) \AA, \angle \mathrm{O}-\mathrm{H} \cdots \mathrm{N} 170.209(18)^{\circ}\right]$ and $\mathrm{C}-\mathrm{Br} \cdots \mathrm{N}[\mathrm{C} \cdots \mathrm{N} 5.1879(6) \AA, \mathrm{Br} \cdots \mathrm{N} 3.3818(4) \AA$, $\left.\angle \mathrm{C}-\mathrm{Br} \cdots \mathrm{N} 160.664(7)^{\circ}\right]$ in complex 1. 


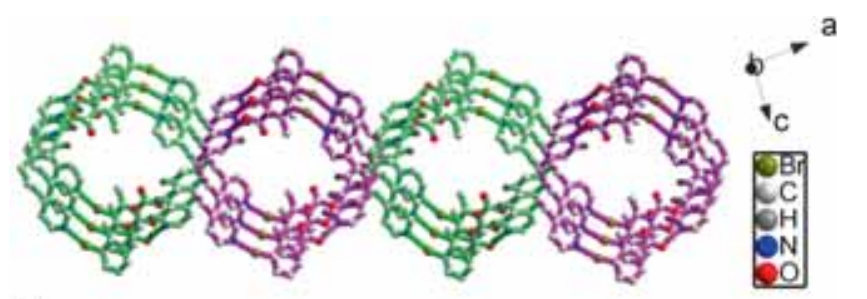

(a)

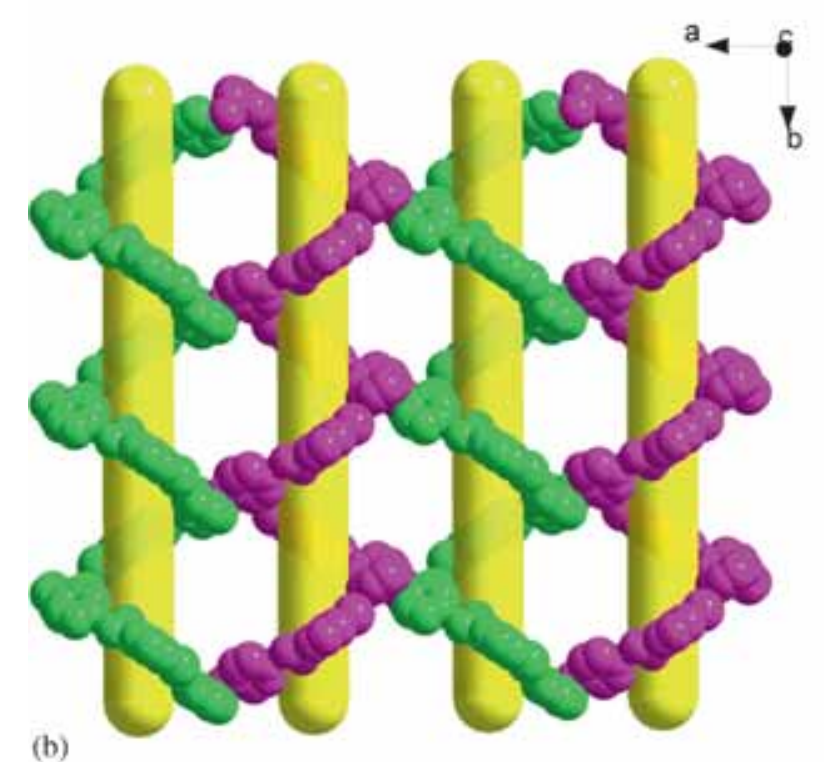

Figure 4. The supramolecular helical architecture of complex 1 viewed from different directions.

Two possible dimers formed by the intermolecular interactions and two related monomers were optimized at the MPW1PW91/6-31G** level (Figure S1). There are no imaginary frequencies for any of the structures, indicating that these structures are indeed the minima on their potential energy surfaces. Some of the fully optimized geometrical parameters together with the experimental data were summarized in Tables S2-S4 (Supplementary Information). It is noted that most of the optimized bond lengths are slightly larger than the experimental values. This may originate from the theoretical calculations which belong to isolated molecules in the gaseous phase at $0 \mathrm{~K}$, while the experimental results of the complex was performed in the solid phase. The deviations in the bond lengths between experimental and calculated geometries are all within $0.03 \AA$ for monomers and dimers I-II, except for the bond C13O1 with a larger discrepancy of $c a .0 .05 \AA$ (Table S2). This is due to $\mathrm{O} 1$ which is involved in forming the strong hydrogen bond $\mathrm{O} 1-\mathrm{H} 1 \mathrm{~A} \ldots \mathrm{N} 1(\mathrm{O} 1 \ldots \mathrm{N} 1$, 2.5908(4) A, Table S4). Similarly, the larger deviations in the bond angles mainly occur on those atoms involved in the hydrogen or halogen bonds, such as O1C13-O2, C16-C17-C18, C15-C16-C17, and C2-C3-C6.
The largest deviation between experimental and theoretical bond angles are $1.154,3.423,3.412$, and $3.513^{\circ}$ for TPB, BMBA and dimers I-II, respectively (Table S3). It can be concluded that precision in the calculations is satisfactory and the MPW1PW91/6$31 \mathrm{G}^{*}$ method is suitable for the supramolecular system studied here.

\subsection{Stability and fluorescence study}

To further demonstrate the stability of the complex 1, thermal gravimetric analysis (TGA) was performed (Figure 5). It was observed that the first weight loss begins at $175^{\circ} \mathrm{C}$. Subsequently, consecutive decompositions take place, leading to the total destruction of the complex. Clearly, it is possible to conclude that the architecture of complex $\mathbf{1}$ is stable within a high temperature region and worthy of further investigation as a candidate of thermally stable material.

At room temperature, excitation of solid samples of complex 1 at $c a .351 \mathrm{~nm}$ produces a strong blue fluorescence emission band at $c a .401 \mathrm{~nm}$ (Figure 6a). To better understand the nature of the luminescent emission, TDDFT calculations were carried out to investigate the electronic transitions in the photoluminescent process of complex $\mathbf{1}$. The results indicate that the lowest energy emission of complex $\mathbf{1}$ is dominated by the transition of LUMO $\rightarrow$ HOMO and assigned to an excited-state intra-ligand charge transfer (ILCT) character. The HOMO mainly consists of $\pi$ orbitals of BMBA, while the LUMO is mostly composed of $\pi^{*}$ orbitals of two pyridine rings of TPB (Figure 6b). The energy gap between HOMO and LUMO is $3.58 \mathrm{eV}$, which agrees reasonably with the experimental value $3.09 \mathrm{eV}(401 \mathrm{~nm})$. The deviation may result from intermolecular interactions such as $\mathrm{HB}$ and $\mathrm{XB}$, which

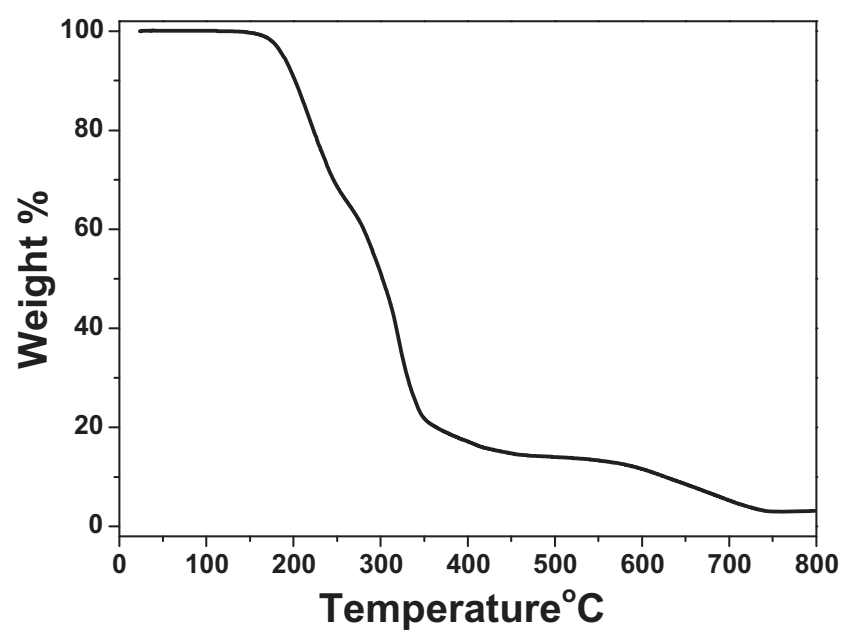

Figure 5. TGA of complex 1. 


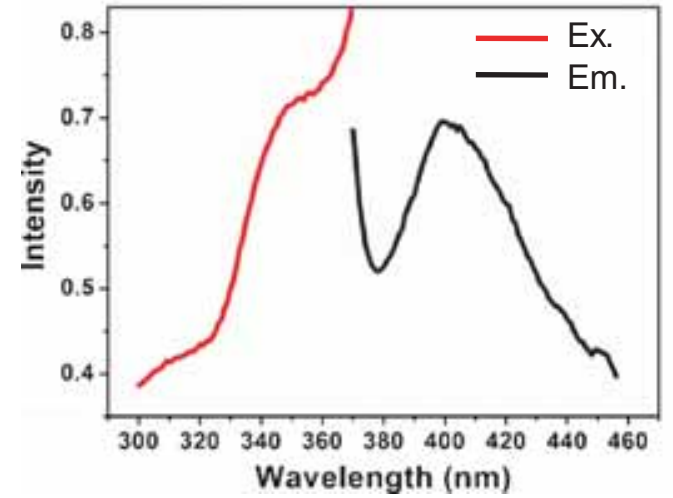

(a)

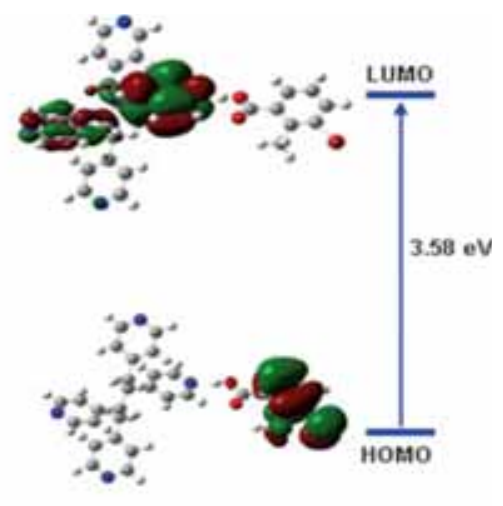

(b)

Figure 6. (a) Fluorescent excitation and emission spectra of $\mathbf{1}$ in the solid state at room temperature. (b) Contour plots of frontier orbitals of $\mathbf{1}$.

play an essential role in decreasing the HOMO-LUMO gap. $^{45}$

\subsection{Interaction energies}

The interaction energies between TPB and BMBA were calculated by MPW1PW91/6-31G** method and listed in Table 1 . The negative value indicates attractive intermolecular interaction, while the positive value indicates repulsive interaction. The results show that even after the BSSE and ZPVE corrections, the total energies of dimers I and II are still smaller than the sum of monomers, implying that the dimers I and II formed by the ligands TPB and BMBA are stable with attractive intermolecular interactions. Also noteworthy is that the corrected interaction energy for the dimer $\mathbf{I}$ is $-44.49 \mathrm{~kJ} / \mathrm{mol}$ and that for the dimer $\mathbf{~ I I}$ is -2.32 $\mathrm{kJ} / \mathrm{mol}$. Because dimers I and II are both connected by only one weak intermolecular interaction (Table S4), the interaction energy can be approximately attributed to the intermolecular hydrogen bond and halogen bond, respectively. Therefore, the binding energy for each hydrogen bond $\mathrm{O} 1-\mathrm{H} 1 \mathrm{~A} . . \mathrm{N} 1$ in dimer $\mathbf{I}$ is $c a .44$ $\mathrm{kJ} / \mathrm{mol}$. This value is of high in comparison with those reported previously $(8-54 \mathrm{~kJ} / \mathrm{mol})^{46}$ and almost as large as thrice the best experimental estimate of the dissociation energy of the water $\operatorname{dimer}(\sim 3 \times 15 \mathrm{~kJ} / \mathrm{mol}),{ }^{47}$ indicating that this hydrogen bond is very strong. In the case of dimer II, the binding energy for each halogen bond $\mathrm{C} 16-\mathrm{Br} 1 \ldots \mathrm{N} 2$ is smaller ( $c a .2 \mathrm{~kJ} / \mathrm{mol})$, showing that the tertiary carbon atom acts as a weak halogen donor. Hence, it is concluded that the hydrogen bonding $\mathrm{O} 1-\mathrm{H} 1 \mathrm{~A} \ldots \mathrm{N} 1$ is far stronger than $\mathrm{C} 16-\mathrm{Br} 1 \ldots \mathrm{N} 2$ and plays a more important role in the crystal packing of the complex 1. Accordingly, the stability of dimers is in the order of $\mathbf{I}>\mathbf{I I}$, which is consistent with that judged from the total electronic energies of dimers $\mathbf{I}$ and II (Table 1).

To determine the appropriateness of the chosen basis set $\left(6-31 \mathrm{G}^{* *}\right)$ for the calculations, the interaction energies have also been calculated at the MPW1PW91/ $6-311++\mathrm{G}^{* *}$ level. The differences in the uncorrected

Table 1. Interaction energies between the monomers calculated at different levels.

\begin{tabular}{|c|c|c|c|c|c|c|c|}
\hline \multirow[b]{2}{*}{ Method } & \multirow[b]{2}{*}{ Molecule. } & \multirow[b]{2}{*}{ E/a.u. } & \multirow{2}{*}{$\begin{array}{c}\mathrm{ZPVE} / \\
\mathrm{kJ} \cdot \mathrm{mol}^{-1}\end{array}$} & \multirow{2}{*}{$\begin{array}{c}\mathrm{BSSE} / \\
\mathrm{kJ} \cdot \mathrm{mol}^{-1}\end{array}$} & \multicolumn{3}{|c|}{ Interaction Energy $/ \mathrm{kJ} \cdot \mathrm{mol}^{-1 c}$} \\
\hline & & & & & $\Delta E$ & $\Delta E_{\mathrm{C}}$ & $\Delta E_{\mathrm{C}, \mathrm{ZPVE}}$ \\
\hline \multirow[t]{4}{*}{ MPW1PW91/6-31G** } & TPB & -1146.5685493 & 1088.3438 & & & & \\
\hline & BMBA & -3031.3033265 & 354.1404 & & & & \\
\hline & Dimer I & -4177.8938081 & 1445.6380 & 10.07 & -57.58 & -47.51 & -44.49 \\
\hline & Dimer II & -4177.8777674 & 1443.6993 & 11.98 & -15.47 & -3.49 & -2.32 \\
\hline \multirow[t]{4}{*}{ MPW1PW91/6-311++G(3df,3pd $)^{b}$} & TPB & -1146.8841185 & 1088.3438 & & & & \\
\hline & BMBA & -3033.8901147 & 354.1404 & & & & \\
\hline & Dimer I & -4180.792244 & 1445.6380 & 0.47 & -47.29 & -46.81 & -43.79 \\
\hline & Dimer II & -4180.7755829 & 1443.6993 & 0.77 & -3.54 & -2.77 & -1.60 \\
\hline
\end{tabular}

${ }^{a} \Delta E$ is the uncorrected interaction energy, $\Delta E_{\mathrm{C}}$ is the interaction energy corrected for BSSE, and $\Delta E_{\mathrm{C}, \mathrm{ZPVE}}$ is the interaction energy corrected for both BSSE and ZPVE, where the scaling factor for ZPVE is $0.96 .{ }^{b} E$ at the MPW1PW91/6$311++\mathrm{G}^{* *} / /$ MPW1PW91/6-31G** level, ZPVE at the MPW1PW91/6-31G** level. 
interaction energies $(\Delta E)$ caused by two different basis sets are at most within $12 \mathrm{~kJ} / \mathrm{mol}$, while those in the interaction energies corrected by $\operatorname{BSSE}\left(\Delta E_{\mathrm{C}}\right)$ are only within $1 \mathrm{~kJ} / \mathrm{mol}$. This indicates that the BSSE correction is necessary for the calculations of $6-31 \mathrm{G}^{* *}$ basis set. On the other hand, both the corrected and uncorrected interaction energies with the $6-311++\mathrm{G}^{* *}$ basis set give the same stability order for the dimers as the $6-31 \mathrm{G}^{* *}$ basis set, although the BSSE computed at the MPW1PW91/6-311++ $\mathrm{G}^{* *}$ level is smaller than that at the MPW1PW91/6-31G* level. This suggests that the $6-31 \mathrm{G}^{* *}$ basis set is suitable and reliable for studying the intermolecular interactions in the title complex.

\subsection{Thermodynamic properties}

In order to elucidate the dimerization process, thermodynamic properties of dimers I, II and related monomers were investigated with the statistical

Table 2. Thermodynamic properties of monomers and dimers at different temperatures ${ }^{a}$.

\begin{tabular}{|c|c|c|c|c|c|c|c|}
\hline Structure & $\mathrm{T} / \mathrm{K}$ & $C_{p, m}^{o} / \mathrm{J} \cdot \mathrm{mol}^{-1} \cdot \mathrm{K}^{-1}$ & $S_{m}^{o} / \mathrm{J} \cdot \mathrm{mol}^{-1} \cdot \mathrm{K}^{-1}$ & $H_{m}^{o} / \mathrm{kJ} \cdot \mathrm{mol}^{-1}$ & $\Delta S_{T} / \mathrm{J} \cdot \mathrm{mol}^{-1} \cdot \mathrm{K}^{-1}$ & $\Delta H_{T} / \mathrm{kJ} \cdot \mathrm{mol}^{-1}$ & $\Delta G_{T} / \mathrm{kJ} \cdot \mathrm{mol}^{-1}$ \\
\hline \multirow[t]{11}{*}{ ТPB } & 200 & 267.01 & 613.72 & 32.52 & & & \\
\hline & 298.15 & 394.86 & 743.63 & 64.87 & & & \\
\hline & 400 & 527.37 & 878.53 & 111.96 & & & \\
\hline & 500 & 637.93 & 1008.50 & 170.41 & & & \\
\hline & 600 & 726.94 & 1132.98 & 238.82 & & & \\
\hline & 700 & 798.35 & 1250.60 & 315.21 & & & \\
\hline & 800 & 856.40 & 1361.12 & 398.05 & & & \\
\hline & 900 & 904.29 & 1464.84 & 486.15 & & & \\
\hline & 1000 & 944.27 & 1562.24 & 578.64 & & & \\
\hline & 1100 & 977.95 & 1653.87 & 674.80 & & & \\
\hline & 1200 & 1006.52 & 1740.22 & 774.06 & & & \\
\hline \multirow[t]{9}{*}{ BMBA } & 200 & 123.82 & 375.94 & 15.51 & & & \\
\hline & 298.15 & 168.53 & 433.77 & 29.88 & & & \\
\hline & 400 & 210.65 & 489.34 & 49.24 & & & \\
\hline & 500 & 244.97 & 540.16 & 72.08 & & & \\
\hline & 600 & 272.50 & 587.35 & 98.01 & & & \\
\hline & 700 & 294.60 & 631.08 & 126.40 & & & \\
\hline & 800 & 312.61 & 671.63 & 156.79 & & & \\
\hline & 900 & 327.51 & 709.33 & 188.82 & & & \\
\hline & 1000 & 340.01 & 744.50 & 222.21 & & & \\
\hline \multirow[t]{11}{*}{ Dimer I } & 200 & 402.06 & 823.21 & 49.09 & -166.45 & -53.50 & -20.21 \\
\hline & 298.15 & 574.07 & 1015.30 & 96.87 & -162.10 & -52.44 & -4.11 \\
\hline & 400 & 749.11 & 1208.95 & 164.42 & -158.92 & -51.34 & 12.23 \\
\hline & 500 & 894.85 & 1392.31 & 246.87 & -156.35 & -50.18 & 28.00 \\
\hline & 600 & 1012.36 & 1566.24 & 342.44 & -154.09 & -48.95 & 43.51 \\
\hline & 700 & 1106.79 & 1729.64 & 448.57 & -152.04 & -47.60 & 58.83 \\
\hline & 800 & 1183.65 & 1882.62 & 563.22 & -150.13 & -46.18 & 73.93 \\
\hline & 900 & 1247.11 & 2025.81 & 684.85 & -148.36 & -44.68 & 88.85 \\
\hline & 1000 & 1300.10 & 2160.03 & 812.29 & -146.71 & -43.12 & 103.59 \\
\hline & 1100 & 1344.75 & 2286.09 & 944.60 & -145.20 & -41.52 & 118.20 \\
\hline & 1200 & 1382.63 & 2404.77 & 1081.02 & -143.77 & -39.88 & 132.65 \\
\hline \multirow[t]{11}{*}{ Dimer II } & 200 & 406.86 & 864.77 & 50.38 & -124.89 & -11.95 & 13.03 \\
\hline & 298.15 & 579.61 & 1058.95 & 98.68 & -118.45 & -10.37 & 24.94 \\
\hline & 400 & 754.37 & 1254.21 & 166.79 & -113.66 & -8.71 & 36.75 \\
\hline & 500 & 899.33 & 1438.66 & 249.72 & -110.00 & -7.07 & 47.93 \\
\hline & 600 & 1015.92 & 1613.33 & 345.70 & -107.00 & -5.43 & 58.77 \\
\hline & 700 & 1109.47 & 1777.22 & 452.14 & -104.46 & -3.77 & 69.35 \\
\hline & 800 & 1185.56 & 1930.50 & 567.02 & -102.25 & -2.12 & 79.68 \\
\hline & 900 & 1248.37 & 2073.88 & 688.81 & -100.29 & -0.46 & 89.80 \\
\hline & 1000 & 1300.86 & 2208.20 & 816.35 & -98.54 & 1.20 & 99.74 \\
\hline & 1100 & 1345.13 & 2334.32 & 948.71 & -96.97 & 2.85 & 109.52 \\
\hline & 1200 & 1382.72 & 2453.02 & 1085.15 & -95.52 & 4.51 & 119.13 \\
\hline
\end{tabular}

${ }^{a} \Delta S_{T}=\left(S_{m}^{o}\right)_{\mathrm{Dimer}}-\left(S_{m}^{o}\right)_{\mathrm{TPB}}-\left(S_{m}^{o}\right)_{\mathrm{BMBA}} ; \Delta H_{T}=\left(H_{m}^{o}+E+\mathrm{ZPVE}\right)_{\mathrm{Dimer}}-\left(H_{m}^{o}+E+\mathrm{ZPVE}\right)_{\mathrm{TPB}}-\left(H_{m}^{o}+E+\mathrm{ZPVE}\right)_{\mathrm{BMBA}} ;$ $\Delta G_{T}=\Delta H_{T}-T \Delta S_{T}$. The scale factor of 0.96 is imposed for frequencies. 
thermodynamics method. ${ }^{33}$ As can be seen from Table 2 , some features were observed. First, thermodynamic functions of dimers and monomers increase with the temperature. This is because of the intensified vibrational movement at a higher temperature which contributes more to the thermodynamic functions, while at a lower temperature the main contributions to the thermodynamic functions come from the translations and rotations of the molecules. The intuitive illustration of the quantitative relationships between the thermodynamic functions and the temperature are as follows (only the dimer I was taken as an example):

$$
\begin{aligned}
& C_{p, m}^{o}=12.06+2.17 T-8.66 \times 10^{-4} T^{2}, \\
& \mathrm{R}^{2}=0.9988, \mathrm{SD}=12.86, P<0.0001 \\
& S_{m}^{o}=393.38+2.23 T-4.62 \times 10^{-4} T^{2}, \\
& \mathrm{R}^{2}=1.0, \mathrm{SD}=1.74, P<0.0001 \\
& H_{m}^{o}=-61.15+0.40 T+4.67 \times 10^{-4} T^{2}, \\
& \mathrm{R}^{2}=0.9996, \mathrm{SD}=8.37, P<0.0001
\end{aligned}
$$

As the temperature increases, the increments for both $C_{p, m}^{o}$ and $S_{m}^{o}$ decrease but that for $H_{m}^{o}$ increases constantly. However, since the coefficients of $T^{2}$ are very small, these correlations approximate to linear equations. In other words, thermodynamic functions of the monomers and dimers increase linearly with the temperature. And the values of $C_{p, m}^{o}, S_{m}^{o}$, and $H_{m}^{o}$ at any temperature can be obtained based on these equations. This may be helpful for further studies on other physical and chemical properties of the title complex.

During the transformation of the monomers to the dimer $\mathbf{I}$, both the entropy and enthalpy changes are negative $\left(\Delta S_{T}<0, \Delta H_{T}<0\right)$ as the temperature increases up to $1200 \mathrm{~K}$, indicating that the dimerization is an exothermic process accompanied by a decrease in the degree of disorder. As for dimer II, the entropy change is also negative at any temperature from 200 to $1200 \mathrm{~K}$, while the enthalpy change becomes positive for the temperature over $900 \mathrm{~K}$. Therefore, the dimerization of II is only an exothermic process for the temperature under $900 \mathrm{~K}$. The order of $\left(\Delta H_{T}\right)_{\mathrm{II}}>\left(\Delta H_{T}\right)_{\mathrm{I}}$ gives the same sequence, $\mathbf{I}>$ II as the binding energies.

Furthermore, during the dimerization processes, the changes of Gibbs free energies $\left(\Delta G_{T}\right)$ are negative for the dimer I under $300 \mathrm{~K}$ while those for II are all positive at any temperature. This suggests that dimer I can be spontaneously produced from the isolated monomers at the room temperature, and the dimerization process for II cannot occur spontaneously. This feature demonstrates that a supramolecular architecture can be assembled via a spontaneous process at the room temperature through the intermolecular hydrogen bond $\mathrm{O} 1-\mathrm{H} 1 \mathrm{~A} \cdots \mathrm{N} 1$ between BMBA and TPB. Judged by the values of $\Delta G_{T}$, the stability order of dimers below $900 \mathrm{~K}$ follows as $\mathbf{I}>\mathbf{I I}$, consistent with that given by the interaction energy. However, above $900 \mathrm{~K}$, the $\Delta G_{T}$ value gives a different stability order $(\mathbf{I I}>\mathbf{I})$. This implies that the halogen bonding $\mathrm{C} 16-\mathrm{Br} 1 \cdots \mathrm{N} 2$ may be more essential for the crystal packing of complex 1 for the temperature above $900 \mathrm{~K}$.

\subsection{Band structure and density of states}

As well known, band gap is an important parameter to characterize the electronic structure of solids. So, based on the experimental crystal structure of the complex 1, the electronic band structure along different symmetry directions of the Brillouin zone was studied (Figure 7). For clarity, only six upper occupied electronic bands (valence bands) and six lower unoccupied electronic bands (conduction bands) were magnified. Both the valence bands and conduction bands are generally quite flat along different symmetry directions of the Brillouin zone. The maximum dispersion the band is $0.05 \mathrm{eV}$ which correspond to the first lowest unoccupied crystalline orbital around $3.29 \mathrm{eV}$. Also noteworthy is that both the valence bands and conduction bands are two-fold degenerate. When small changes in charge state occur, some orbitals are stabilized and others are destabilized, which eliminates the degeneracy of occupied crystalline orbitals and unoccupied crystalline orbitals which could lead to minor JahnTeller distortions. ${ }^{48}$ The energy gap between the highest occupied crystalline orbital (HOCO) and the lowest

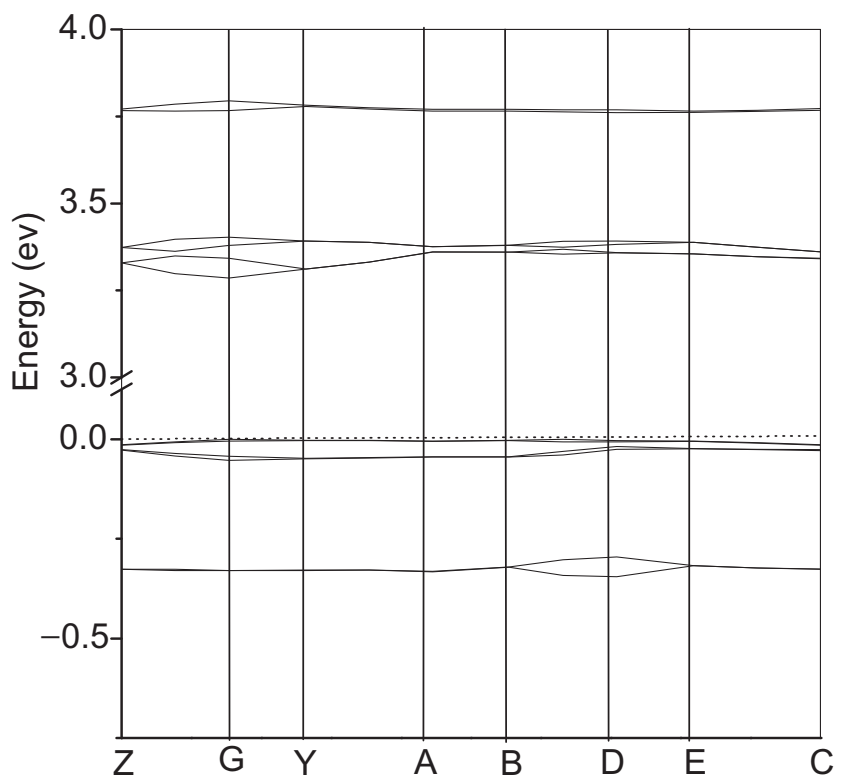

Figure 7. Band structure of the complex 1 along different symmetry directions of the Brillouin zone. The Fermi energy is shown as a dashed horizontal line. 
unoccupied crystalline orbital (LUCO) for the title complex is $3.29 \mathrm{eV}$, indicating that it is an electrical insulator. Compared with the experiment results, the band gaps are always underestimated by DFT calculations. And the real band gap may be slightly larger these calculated results.

The total electronic density of states (DOS) was also calculated to obtain more information about the bonding nature of complex $\mathbf{1}$. In the upper valence band, the crystal has a sharp peak near the Fermi level, which shows that the top valence band of the band structure is flat (Figure 8). The peak is predominately from the $p$ states. After that, several main peaks in the upper valence band are superimposed by the $s$ and $p$ states. The conduction band is dominated by the $p$ states. These all indicate that the $p$ states play an important role in the chemical reactions of the title complex.

A better understanding of the band character can be further examined directly by projecting the DOS on the atom-centered orbitals (Figure 8). It can be seen that in the upper valence bands close to the Fermi level, the $p$ states of $\mathrm{C}, \mathrm{N}, \mathrm{O}$ in $-\mathrm{C}=\mathrm{O}$, and $\mathrm{Br}$ atoms make more important contributions than those of the atoms $\mathrm{H}$ and

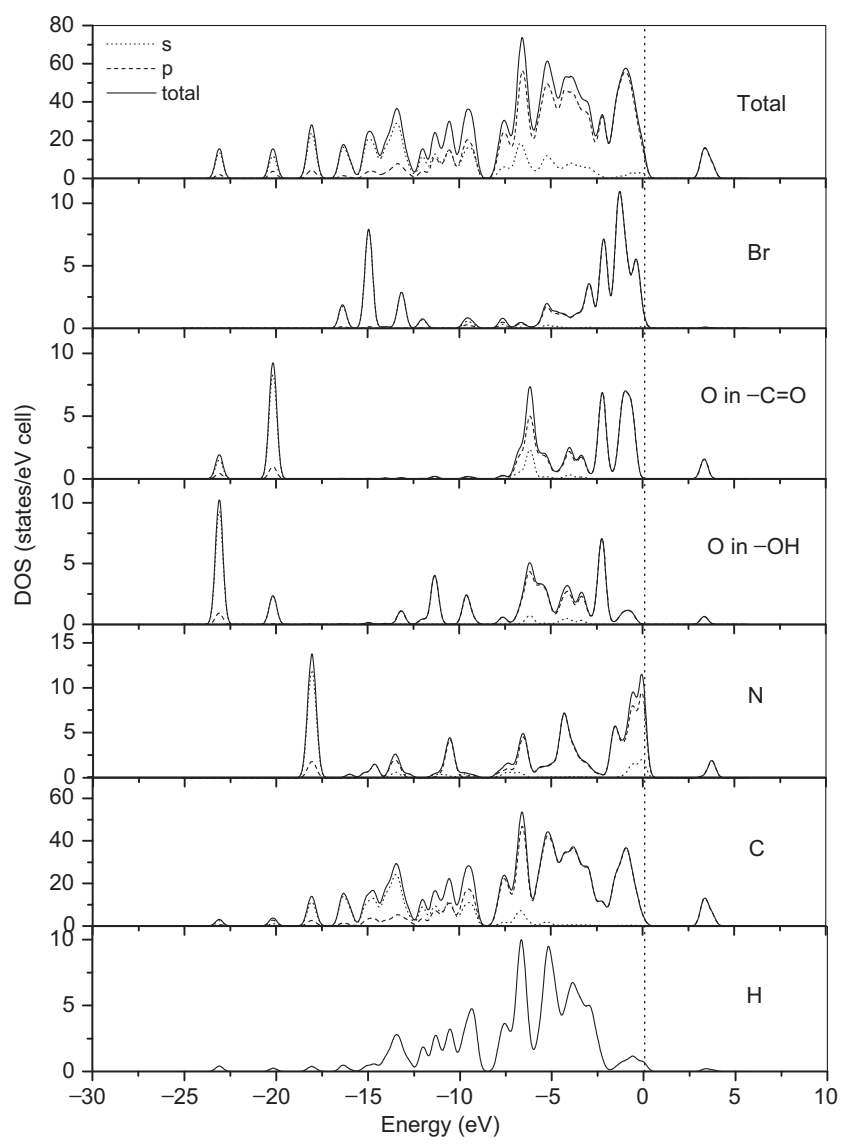

Figure 8. Total and partial density of states (DOS) of the complex 1. The Fermi energy is shown as a dashed vertical line.
$\mathrm{O}$ in $-\mathrm{OH}$. It is hence expected that the upper valence bands mainly consist of the $\mathrm{C}, \mathrm{N}, \mathrm{O}$ in $-\mathrm{C}=\mathrm{O}$, and $\mathrm{Br}$ atoms. In contrast, the conduction bands are dominated by the $p$ states of $\mathrm{C}, \mathrm{N}$ and $\mathrm{O}$ atoms. Therefore, the ring atoms of $\mathrm{C}$ and $\mathrm{N}$ are mixed up in the six-member ring which is consistent with the analytical result from the determined crystal structure.

\section{Conclusions}

An interesting 2D helical supramolecular architecture driven by hydrogen and halogen bonding was synthesized and characterized. Experimental results have been fully rationalized at the molecular level via DFT and TDDFT calculations. The cooperation between HB and $\mathrm{XB}$ in building up open organic architectures was successfully demonstrated, although the former is far stronger than the latter. At room temperature, the dimerization of TPB and BMBA is an exothermic process accompanied by a decrease in the degree of disorder, and a supramolecular architecture can be assembled via a spontaneous process through the intermolecular hydrogen bonding $\mathrm{O}-\mathrm{H} \cdots \mathrm{N}$. Thermogravimetric analysis and luminescence spectroscopy indicate that complex $\mathbf{1}$ is a potential candidate of thermally stable blue fluorescent materials with the intra-ligand charge transfer character. Band structure and density of states calculations show that the crystal of complex $\mathbf{1}$ is an electrical insulator with a band gap of $3.29 \mathrm{eV}$.

\section{Supplementary Information}

CCDC 758843 contains supplementary crystallogra phic data of complex 1 . The data can be obtained free of charge via http://www.ccdc.cam.ac.uk/conts/retrieving. html (or from the Cambridge Crystallographic Data Centre, 12, Union Road, Cambridge CB2 1EZ, UK; fax: t44 1223 336033) or e-mail: deposit@ccdc.cam.ac.uk. Figures S1 and S2, and the details of crystal parameters, data collection and refinements for the complex $\mathbf{1}$ are summarized in Table S1 in Supporting Information. Selected bond lengths and angles are listed in Tables S2-S4. Supplementary Information is available at www.ias.ac.in/chemsci.

\section{Acknowledgements}

The authors appreciate the financial support from National Natural Science Foundation of China (2137 1082), Natural Science Foundation of Jiangsu Province (BK20151118, BK20141102), and Key Medical Talent Project of Jiangsu Province (RC2011097). 


\section{References}

1. Keeling D L, Oxtoby N S, Wilson C, Humphry M J, Champness N R and Beton P H 1994 Nano Lett. 39

2. Zhang Y, Anderson C A and Zimmerman S C 2013 Org. Lett. 153506

3. Meazza L, Foster J A, Fucke K, Metrangolo P, Resnati G and Steed J W 2013 Nat. Chem. 542

4. Priimagi A, Cavallo G, Metrangolo P and Resnati G 2013 Acc. Chem. Res. 462686

5. Sharma A, Harnish P, Sylvester A, Kotov V N and Neto A H C 2014 Phys. Rev. B 89235425

6. Jeffrey G A 1997 In An Introduction to Hydrogen Bonding (New York: Oxford University Press)

7. Politzer P, Lane P, Concha M C, Ma Y and Murray J S 2007 J. Mol. Model. 13305

8. Voth A R and Ho P S 2007 Curr. Top. Med. Chem. 7 1336

9. Metrangolo P, Meyer F, Pilati T, Resnati G and Terraneo G 2008 Angew. Chem. Int. Ed. 476114

10. Mocilac P and Gallagher J F 2014 CrystEngComm 16 1893

11. Kaur D and Kaur R 2014 J. Chem. Sci. 1261763

12. Metrangolo P, Neukirch H, Pilati T and Resnati G 2005 Acc. Chem. Res. 38386

13. Bouchmella K, Boury B, Dutremez S G and van der Lee A 2007 Chem. Eur. J. 136130

14. Mukherjee A 2015 Cryst. Growth Des. 153076

15. You L Y, Chen S G, Zhao X, Liu Y, Lan W X, Zhang Y, Lu H J, Cao C Y and Li Z T 2012 Angew. Chem. Int. Ed. 511657

16. Tothadi S and Desiraju G R 2013 Chem. Commun. 49 7791

17. Awwadi F F, Taher D, Haddad S F and Turnbull M M 2014 Cryst. Growth Des. 141961

18. Yan X C, Schyman P and Jorgensen W L 2014 J. Phys. Chem. A 1182820

19. Xiao H M, Ju X H, Xu L N and Fang G Y 2004 J. Chem. Phys. 12112523

20. Qiu L, Li W X, Fan X W, Ju X H, Cao G X and Luo S N 2008 Inorg. Chem. Commun. 11727

21. Brela M, Stare J, Pirc G, Sollner-Dolenc M, Boczar M, Wójcik M J and Mavri J 2012 J. Phys. Chem. B 1164510

22. Yu L J, Pang R, Tao S, Yang H T, Wu D Y and Tian Z Q 2013 J. Phys. Chem. A 1174286

23. Qiu L, Liu Q Z, Wang Y, Wang T F, Yang H, Ju X H, Luo S N and Lin J G 2015 Struct. Chem. 26845
24. Hünig S, Langels A, Schmittel $M$, Wenner $H$, Perepichka I F and Peters K 2001 Eur. J. Org. Chem. 20011393

25. Thayer H I and Corson B B 1948 J. Am. Chem. Soc. 70 2330

26. SAINT. Program for Data Reduction from Area Detectors (Madison WI: BRUKER AXS Inc.)

27. Sheldrick G M 2008 SADABS (Germany: University of Göttingen)

28. Sheldrick G M 1997 SHELXS97 (Göttingen Germany: University of Göttingen)

29. Sheldrick G M 1997 SHELXL97 (Göttingen Germany: University of Göttingen)

30. Adamo C and Barone V 1998 J. Chem. Phys. 108664

31. Hariharan P C and Pople J A 1973 Theo. Chem. Acc. 28 213

32. Scott A P and Radom L 1996 J Phys. Chem. 10016502

33. Hill T L 1960 In Introduction to Statistical Thermodynamics (New York: Addision-Wesley)

34. Boys S F and Bernardi F 1970 Mol. Phys. 19553

35. Krishnan R, Binkley J S, Seeger R and Pople J A 1980 J. Chem. Phys. 72650

36. McLean A D and Chandler G S 1980 J. Chem. Phys. 72 5639

37. Frisch M J et al. 2009 Gaussian 09, Revision A.02 (Wallingford CT: Gaussian Inc.)

38. Berski S, Ciunik Z, Drabent K, Latajka Z and Panek J 2004 J. Phys. Chem. B 10812327

39. Vanderbilt D 1990 Phys. B 417892

40. Segall M D, Philip J D L, Probert M J, Pickard C J, Hasnip P J, Clark S J and Payne M C 2002 J. Phys: Condens. Matter. 142717

41. Perdew J P, Burke K and Ernzerhof M 1996 Phys. Rev. Lett. 773865

42. Kresse G and Furthmüller J 1996 Phys. Rev. B 5411169

43. Monkhorst H J and Pack J D 1976 Phys. Rev. B 135188

44. Bondi A 1964 J. Phys. Chem. 68441

45. Zheng S L, Zhang J P, Wong W T and Chen X M 2003 J. Am. Chem. Soc. 1256882

46. Philp D and Stoddart J F 1996 Angew. Chem. Int. Ed. 35 1154

47. Feyereisen M W, Feller D and Dixon D A 1996 J. Phys. Chem. 1002993

48. Hoffmann R 1988 In Solids and Surfaces: A Chemist's View of Bonding in the Extended Structures (New York: $\mathrm{VCH})$ 